\title{
PENSAMIENTO CRÍTICO Y LECTURA EN CIENCIAS SOCIALES
}

\section{CRITICAL THINKING AND READING IN SOCIAL SCIENCES}

\author{
Jesús, A. Morales-Carrero ${ }^{1}$ \\ jesusm100386@gmail.com \\ Universidad de Los Andes, Venezuela
}

\section{Volumen 8, Número 2}

30 de noviembre de 2017

pp. $265-282$

Recibido: 15 de mayo de 2017

Aprobado: 2 de noviembre de 2017

\footnotetext{
${ }^{1}$ Politólogo y Abogado egresado la Universidad de Los Andes. Magister en Educación mención Orientación Educativa por la Universidad Pedagógica Experimental Libertador. Docente en la categoría Instructor de la Escuela de Criminología ULA en las asignaturas: Desarrollo personal, Método científico, Técnicas de estudio y Lectoescritura y metodología del estudio en la Modalidad de Derecho Interactivo a Distancia (EIDIS). Correos electrónicos: jesusm100386@gmail.com/ lectoescrituraula@gmail.com
} 


\section{Resumen}

La apreciación de la lectura como una actividad social, se ha posicionado como una de las maneras de potenciar el desarrollo del pensamiento y en especial un pensamiento crítico y reflexivo (Lipman, 1998). De allí que el propósito del presente artículo consista en realizar una disertación sobre las competencias y habilidades cognitivas que debe manejar el lector de las ciencias sociales, en el siglo XXI,al momento de abordar los géneros propios de su área (Cassany, 2004). Por ende, se espera entonces que el lector asuma un posicionamiento crítico, una actitud consciente responsable y autónoma como condiciones que lo autoricen para cuestionar el saber y así construir una voz propia que refleje su compromiso autocrítico frente al conocimiento y frente a la transformación de la realidad social (Sanmarti, 2011).

Palabras clave: Lectura crítica, ciencias sociales, pensamiento crítico, habilidades cognitivas, conocimiento científico.

\section{Abstract}

The appreciation of reading as a social activity, has positioned itself as one of the ways to foster the development of thought and especially a critical and reflective thinking (Lipman, 1998). Hence the purpose of this article is to make a dissertation about the cognitive competences and skills that the reader of the social sciences should handle in the 21 st century when addressing their own gender area (Cassany, 2004). Therefore, the reader is expected to assume a critical position, a responsible and autonomous conscious attitude like conditions that authorize him to question the knowledge and thus to construct one own voice that reflects his self-critical commitment to knowledge and against the transformation of social reality (Sanmarti, 2011).

Keywords: Critical reading, social sciences, critical thinking, cognitive abilities, scientific knowledge. 


\section{INTRODUCCIÓN}

La lectura como una actividad social, ha sido asumida desde los diversos escenarios académicos y científicos como la manera por excelencia de potenciar el desarrollo del pensamiento y en especial el pensamiento crítico. Por ende, la consigna de una educación para y a lo largo de la vida (Torres, 2006) se ha posicionado como el lema que entraña la necesidad de aprender y adaptarse a las múltiples maneras como la información se presenta en el presente siglo. Por ello, la recurrente e indiscutible demanda de un pensamiento educado que funcione de manera acuciosa y que le permita al ciudadano dilucidar las finalidades y múltiples objetivos subyacentes, las ideologías e ideas oscuras y, a las pretensiones, se ha convertido en una necesidad para actuar frente a un discurso complejizado por la abundante información derivada de comunidad científica, es decir, de sus prácticas y formas de producir y presentar el conocimiento (Cassany, 2004; Díaz, Bar y Ortiz, 2015; Morín, 2007; Santiuste, 2001).

Esta visión de la lectura, como un proceso dinámico y de la realidad como espacio de transformación constante, ha sido vista como una herramienta al servicio de la formación del pensamiento, pues entre sus bondades se encuentran el favorecer el acercamiento al conocimiento al propiciar interacciones efectivas con ideas y datos informativos, que sometidos a la rigurosidad de la criticidad y la reflexión, le permite el dar cuenta de situaciones, hechos y acontecimientos de los que el lector consiga elaborar apreciaciones sólidas y sustentadas que asumidas como premisas lógicas y coherentes, aportar apreciaciones sobre el mundo y su contexto, como expresiones (Aisenberg, 2007; Carlino, 2005; Sanmartí, 2011) que lo lleven trascender, a transformar y por ende aportarle alternativas de cambio a su espacio de convivencia inmediata (Cassany, 2004; Serna y Díaz, 2015).

Pensamiento Crítico y Lectura en Ciencias Sociales

Jesús A. Morales-Carrero

DOI : http://dx.doi.org/10.22458/caes.v8i2.1943

\section{(c) ${ }_{\mathrm{BY}}($ SC}


Visto esto desde el aprendizaje de la lectura en función de la perspectiva sociocultural como la tendencia más reciente, se intenta acercar al lector a un procesamiento eficiente de información, al exigirle entre otras condiciones que fije posición crítica, responsable y autónoma (Cassany, 2004; 2006) como habilidades que le cooperen en el proceso de valoración contenidos, la puesta a prueba de su veracidad, pero que además que consiga evaluar los datos objetivamente (Carlino, 2003). Bajo este cúmulo de exigencias académicas, se pretenden con la presente disertación, realizar un acercamiento a las bondades del pensamiento crítico para formar lectores reflexivos en ciencias sociales (Bartz, 2002; Jurado, 2008; Kurland, 2003) capaces de releer, seleccionar, objetar posturas, identificar argumentos en pro y en contra, relacionar, interpretar, de fijar una postura frente al saber histórico (Aisenberg, 2007; Hawes, 2003; Páges, 2009) en un afán de conseguir, como lo expone Peppino (2006), llegar a "reflexionar sobre la importancia, la veracidad y aportación de lo que se ha leído" (p.1).

\section{PENSAMIENTO CRÍTICO Y LA LECTURA EN CIENCIAS SOCIALES}

Aprender a pensar y a leer de manera crítica en las diferentes etapas de la educación y en específico en un área tan importante como ciencias sociales, se ha precisado como un objetivo a su vez un reto complejo y difícil de desarrollar (Carlino, 2011; Delors, 1996; Lerner, Aisenberg y Espinoza, 2011; Morín, 2007; Reale, 2016) debido fundamentalmente a la coexistencia de visiones reduccionistas derivadas de una educación maniquea, cuyo punto focal ha sido la enseñanza limitada a una óptica o postura de los hechos y acontecimientos, dejando de atender la multiplicidad de aristas y enfoques desde los cuales aproximarnos a la comprensión del contexto y del momento estudiado. Por ende, la frecuente asunción de una tendencia ideológica y la familiarización hacia determinados postulados (autores) más que a otros, ha

Pensamiento Crítico y Lectura en Ciencias Sociales

Jesús A. Morales-Carrero

DOI : http://dx.doi.org/10.22458/caes.v8i2.1943

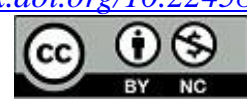


imposibilitado la generación de posturas críticas y reflexivas que expongan objetivamente apreciaciones sobre el saber dando espacio a la pluralidad de opiniones.

En este sentido, el estudio de las ciencias sociales como un campo abierto a la existencia de posiciones ideológicas diversas, ha entrañado visiones sesgadas e incompletas (Becker, 2011) en las que la incomprensión y la distorsión han reinado en función de los intereses de terceros. Frente a ello, se han esgrimido planteamientos que exigen, que la lectura como actividad social, deba permitir la convivencia y discusión de las diversas miradas y aristas pronunciadas, registradas y mostradas sobre los productos del actuar humano, favoreciendo la manifestación de habilidades cognitivas que le permitan al ciudadano en formación exponer, explicar y argumentar sobre las visiones aisladas y carentes de significado y manipuladas que solo llevan a la construcción de conocimiento errado, subjetivo y a la elaboración de apreciaciones mediadas por la criticidad como condición fundamental para acercarse al mundo del leer en ciencias sociales (Carlino, 2006; Cassany, 2006; Serrano, 2008; Pérez, 2005; Peppino, 2006).

Asimismo, el pensar y leer de manera crítica contenidos tan controversiales y complejos como los de ciencias sociales, exige del lector una actitud acuciosa caracterizada por una constante y recurrente revisión de posturas teóricas y de un pensamiento agudo, que le permita valorar los argumentos de forma cuidadosa y minuciosa, a fin de determinar la validez del conocimiento, con el afán de dilucidar sus propósitos, la detección de aspectos débiles y refutables que, aún y cuando vienen respaldadas por autoridades con reconocimiento científico (cualidad que los reviste de legitimidad) pueden ser falibles, a lo que el lector con un pensamiento crítico debe aplicar la rigurosidad necesaria para objetar su falsedad a través del uso de los criterios de veracidad, pertinencia y validez como resultado su criticidad (Becker, 2011).

Pensamiento Crítico y Lectura en Ciencias Sociales

Jesús A. Morales-Carrero

DOI : http://dx.doi.org/10.22458/caes.v8i2.1943 
De esto se puede inferir, que el leer en un siglo tan saturado de información emanada de diversas fuentes académicas y no académicas, representa un reto para los que se forman en la actualidad. Ello debido fundamentalmente a que las demandas a las que se ve enfrentado el lector como un agente activo y enmarcado en un contexto en permanente transformación, pasa por exigirle el despliegue de operaciones mentales que le conduzcan a reconocer no solo la autoría, la autoridad en los textos y las fuentes, sino además, el uso de la criticidad como una manera de refutar, de objetar planteamientos y de contraargumentar ideas y afirmaciones, en un afán de dar cuenta del uso del pensamiento crítico y reflexivo (Carrasco y Kent, 2011; Lipman, 1997) como cualidad que debe caracterizar a todo ciudadano consiente y comprometido con lo social (González y Vega, 2010).

Por tal motivo, desde la lectura en las disciplinas (Díaz, 2015) han emergido sugerencias importantes para atender las particularidades de los textos, entre ellas se resaltan la consideración de los siguientes aspectos: identificación de la autoría, postura ideológica y científica y, cómo se organiza sus aportes (ensayos, monografías, artículos e informes) sin dejar de atender a la estructura de cada género, familiarizarse con la presentación interna y externa, pero además, el desplegar habilidades cognitivas como la predicción y la inferencia, así como la intertextualidad, en la que el lector se apoye y lo aproximen con mayor claridad, como condiciones y exigencias propias para procesar y apropiarse coherentemente del conocimiento (Goodman, 1996; Jurado, 2008; Olson, 1994; Reale, 2016).

Todas estas demandas que demuestran las implicaciones de la lectura académica de textos de las ciencias sociales, representan razón suficiente para que el lector ponga a prueba sus competencias para deconstruir como proceso que le lleve a con-construir conocimiento, es decir, que como sujeto crítico debe responder a demandas que le requieren una interacción con los textos, con su terminología y con las formalidades, pero además con los propósitos y

Pensamiento Crítico y Lectura en Ciencias Sociales

Jesús A. Morales-Carrero

DOI : http://dx.doi.org/10.22458/caes.v8i2.1943 
pretensiones que los autores dejan a criterio del lector (2011) esperando que este logre desentrañar lo que subyace, lo que no está a simple vista, pero que tiene un valor significativo para generar procesos de apropiación efectiva del conocimiento. A ello se agrega lo propuesto por Sanmartí (2011), quien afirma que esta forma de hacer lectura implica entre otras cosas el "ser capaces de modificar conocimientos y de apropiarse de nuevos formatos y maneras a lo largo de su vida y esta competencia comporta ser capaz de leer todo de manera autónoma, significativa y crítica los distintos tipos de textos" (p.2).

Por lo tanto, es en este marco de exigencias implicadas en el leer en ciencias, que se logran precisar un conjunto de expectativas que posicionan como imperante la necesidad de educar al pensamiento hacia niveles superiores. Lo que indefectiblemente requiere de un lector capaz de aplicar estrategias y habilidades cognitivas que lo lleven de acuerdo al planteamiento de Reale (2016) a "analizar e interpretar críticamente la información, distinguir, sostener y refutar diferentes puntos de vista en torno de un objeto e investigar en distintos tipos de fuentes" (p.9). De esta afirmación, es importante precisar lo siguiente, el leer en ciencias sociales, además de permitir el valorar la veracidad de la información expuesta en los textos, también representa una herramienta de aprendizaje disciplinar, que le permite al lector el asumir con responsabilidad posicionamientos autónomos y decidir en función de aspectos como la veracidad y el rigor científico como criterios que permitan configurar interpretaciones diversas sobre problemas o situaciones propios de su contexto.

Ahora bien, desde apreciaciones epistemológicas leer y pensar desde las ciencias sociales requiere además de un pensamiento evaluador que objete lo sucedido en la realidad y en las fijaciones teóricas dadas desde el mundo científico, atendiendo desde una visión sistemática su comprensión, es decir, que consiga generar articulaciones que relacionen fuentes de producción de conocimiento, su evolución y transformaciones a lo largo del tiempo, así como

Pensamiento Crítico y Lectura en Ciencias Sociales

Jesús A. Morales-Carrero

DOI : http://dx.doi.org/10.22458/caes.v8i2.1943 
las derivaciones teóricas y su estructuración como resultado de convenciones científicas propias de cada disciplina (Hammer, 1994). Por consiguiente, se debe entender el acercamiento al conocimiento científico como un proceso que visto desde múltiples aristas, intenta llevar al lector a la consolidación de ideas sólidas, fundadas en la objetividad y en la rigurosidad como resultado de una actitud crítica y reflexiva precisa que sea capaz de generar modificaciones y explicaciones novedosas a partir del manejo de sus premisas (Smith y Anderson, 1993).

En atención a estos planteamientos Santelices (s/f) habla que la lectura dentro de las asignaturas científicas debe ser capaz de propiciar el desarrollo de habilidades, entre las cuales destaca "descubrir problemas, contradicciones, limitaciones y argumentos que apoyen o refuten determinadas ideas o planteamientos y decidir racionalmente qué hacer o creer" (p.1). Desde esta perspectiva, la lectura puede ser entendida como un proceso fundado en la toma de decisiones, en las que el lector de manera recursiva va sobre el texto bien sea para explorar superficialmente, o para criticar y evaluar la información, acudiendo al raciocinio como una habilidad superior que le permita dilucidar las inconsistencias, aspectos oscuros 0 confusos a los cuales explicitar, asumiendo para ello dos alternativas, por un lado, adherirse y aceptar, o por el contrario objetar con premisas objetivas y lógicas (Lerner, 2011).

En este mismo orden de ideas, Peppino (2006), quien hace referencia a este compromiso crítico y reflexivo que reviste el leer académicamente, propone que, se requiere de madurez para ver en la lectura un medio poderoso de acceso al conocimiento, que favorece el consolidar estructuras conceptuales de solido sustento como indicadores que coadyuvan a tomar decisiones con un amplio sentido de responsabilidad. Por consiguiente que "la lectura académica permite discriminar lo útil de lo inútil, lo falso de lo comprobable, lo superfluo de lo necesario y, también, permite evaluar el propio conocimiento y la necesidad, dado el caso, de

Pensamiento Crítico y Lectura en Ciencias Sociales

Jesús A. Morales-Carrero

DOI : http://dx.doi.org/10.22458/caes.v8i2.1943 
ampliarlo o profundizarlo" (p.1). Este enfoque afirma que, el acceso al discurso académico amerita de la activación de operaciones mentales que permitan al lector organizar y movilizar sus conocimientos para trascender de la mera evaluación de contenidos hacia el problematizar ideas, detectar argumentos y tipificarlos, deducir razonamientos y conclusiones en virtud de fijar posición crítica frente al conocimiento (Becker, 2011; Cassany, 2006; Carlino, 2006; Sanmarti, 2011; Serrano, 2008).

No obstante, el rol del lector como agente crítico y estudioso de la realidad, requiere del asumir con responsabilidad (Cassany, 2006) la detección de apreciaciones críticas como resultado de una revisión exhaustiva de pistas útiles o claves sugeridas, en las cuales el lector se apoye para conseguir puntos referenciales que lo lleven a establecer comparaciones y analogías que le permitan dilucidar las pretensiones reales del autor (Reale, 2016) y las miradas subyacentes como muestra de una valoración reflexiva, pero que ponga de manifiesto los posicionamientos ideológicos y matices teóricos que puedan estar permeando la información (Becker, 2011) hasta trascender a construcciones pertinentes y significativas para el conocimiento disciplinar (Sanmartí, 2011).

En razón de ello, el lector en su quehacer científico dentro de las ciencias sociales, debe valerse de habilidades cognitivas que le cooperen con la elaboración de interrogantes y acercamientos interpretativos que lo lleven a conseguir progresivamente sentido en los textos, apropiándose de formas discursivas, usos y funciones sociales (Aisenberg, 2007). Conviene subrayar, entonces que, a la luz de los postulados y referentes de la lectura y pensamiento crítico se pretende que el lector pueda abrirse espacios que motiven la participación y la elaboración conjunta de apreciaciones y opiniones, que una vez contrastadas con otros textos más complejos funjan como referentes para valorar, verificar y aplicar lo leído a la comprensión

Pensamiento Crítico y Lectura en Ciencias Sociales

Jesús A. Morales-Carrero

DOI : http://dx.doi.org/10.22458/caes.v8i2.1943

\section{(c) ${ }_{\mathrm{BY}}($ SC}


de la realidad, en un sentido más amplio, llegar a la modificación de su entorno como resultado de un pensamiento superior (Kock, 1991).

Con todo lo anterior, es posible afirmar que, desde la lectura crítica como aliada del lector de las ciencias sociales, se hace especial énfasis en la necesidad de conocer cómo tiende a organizarse el discurso dentro del área, pues es muy frecuente que los autores acudan a artimañas evasivas como resultado de un juego terminológico que entraña aparentes nociones sobre puntos medulares que explican el tema. Por ese motivo, es indispensable entender que los escritores en ciencias sociales responden a una manera particular de mostrar el conocimiento, que de acuerdo al discernimiento de Becker (2011), consiste en "llevar al lector a que crea todo, hasta que llega al último párrafo, por demás impactante, que sintetiza el argumento y las pruebas" (p. 38). Es allí, donde el lector debe desplegar las bondades del pensamiento crítico, al detectar como parte de sus funciones los fundamentos sobre los que se sostienen las ideas, los planteamientos ideológicos de terceros, sus tesis y argumentos, que una vez sometidos a la verificación, al reconocimiento de sus componentes y a la rigurosidad científica permiten determinar su fiabilidad (Aisenberg, 2007; Peppino, 2006; Sanmarti, 2011; Ulloa, Crispín y Béjar, s/f).

En esta misma línea de pensamiento, Cassany (2006) propone que leer desde la perspectiva crítica implica "la adquisición de destrezas cognitivas que permitan el detectar las intenciones del autor, extraer el contenido que aporta un texto y verificar si es correcto o no" (p.82). En razón de ello, el rol del lector debe estar centrado en el llegar un grado tal de profundización en las ideas y planteamientos de terceros, que le permita identificar con ello los aspectos medulares y la certeza de sus afirmaciones y datos, en una constante que va entre la búsqueda de verificación y la comprobación de su veracidad. Esto indica que, el lector al contar con un arsenal cognitivo agudizado, está posibilitado para conseguir con mayor fluidez y precisión el

Pensamiento Crítico y Lectura en Ciencias Sociales

Jesús A. Morales-Carrero

DOI : http://dx.doi.org/10.22458/caes.v8i2.1943 
establecer redes de interacción con otros textos y posturas que lo doten y autoricen para emitir juicios e interpretaciones (Fortes y Lomnitz, 1991; Hawes, 2003; Sanmarti, 2011).

Como consecuencia, es posible afirmar que el leer y pensar críticamente, consiste también en procesar, falsear o confirmar en función del criterio "credibilidad" los postulados esgrimidos en los textos científicos, para lo cual se ha considerado ineludible que el lector se valga entre otros aspectos de la revisión del aspecto "autoridad" que ostenta el autor dentro de la comunidad científica a la que pertenece, su línea de investigación, quienes validan y reconocen sus producciones, así como la pertinencia de sus apreciaciones. Aunado a ello, algunos especialistas sugieren la necesidad de someter a la rigurosidad de operaciones superiores como el análisis de los componentes de sus argumentos y planteamientos, es decir, la información sobre la que sostiene sus premisas, las fuentes que usa, las citas y referencias usadas para apoyar o asumir una u otra posición (Bazerman, 1988; Carrasco y Kent, 2011).

En acuerdo con Peppino (2006), pensar de manera crítica al momento de hacer lectura académica consiste en "ir más allá de la comprensión de lo que se lee, es identificar ideas y opiniones, hasta conseguir argumentos para justificar la aceptación de las mismas" (p.2). Esta forma de interactuar con los textos implica entonces, que el autor es sometido a una evaluación exhaustiva en un intento por precisar lo que subyace en su pensamiento, sus visiones y posturas ideológicas, sus creencias y afirmaciones que da por ciertos o que refuta, en una búsqueda de razonamientos válidos que comprueben la calidad y solidez de su manera de ver la realidad, de concebir el mundo, pero además de cómo lo contextualiza para que el lector lo pueda utilizar (Cassany, 2009; Sanmartí, 2011).

De atención a este cúmulo de exigencias derivadas del leer en ciencias sociales, es oportuno proponer algunas interrogantes que todo lector debe considerar para adentrarse en el

Pensamiento Crítico y Lectura en Ciencias Sociales

Jesús A. Morales-Carrero

DOI : http://dx.doi.org/10.22458/caes.v8i2.1943 
conocimiento disciplinar y en el desarrollar el pensamiento crítico. Después de esta exposición sumaria, se traen a colación algunas ideas propuestas; Argudin y Luna (1995) junto a Smith (1983), Goodman (1996) y Solé (1996) dejan por sentado algunos aspectos que un lector crítico de las ciencias sociales debe poseer, entre ellos se mencionan: ¿Cuál es la fuente? ¿Es veraz? ¿Está actualizada? ¿Cuál es el propósito y objetivo del autor? ¿Cómo presenta el autor la información? (hechos, inferencias u opiniones) ¿qué tono utiliza el autor? ¿Qué lenguaje utiliza el autor? ¿Cuál es la hipótesis o tesis que el autor propone? ¿Es coherente y sólida la argumentación del autor? ¿Cambió mi opinión el texto? ¿Me hizo reflexionar? (Argudin y Luna, 1995; Cassany, 2006; Lerner, 2011; Smith; 1983, Goodman, 1996; Reale, 2016; Solé, 1996).

En definitiva, se trata de que el lector asuma con responsabilidad el manejo y uso de los nuevos significados que utilizados en situaciones comunicativas, entiéndase escritura disciplinar como vehículo para expresar el conocimiento, pueda dar cuenta de la rigurosidad de su pensamiento crítico en una apropiación consciente de sus prácticas y convenciones que una vez interiorizadas le permiten acceder a mayores niveles de comprensión y a la construcción de una voz propia que dé cuenta de su manera de concebir el conocimiento (Hawes, 2003; Lipman, 1998; Newmann, 1990; Serna y Díaz, 2015).

\section{CONCLUSIONES}

Como se puede observar, las apreciaciones derivadas de la revisión teórica sobre lo que implica leer y pensar de manera crítica, demuestran que en el campo de las ciencias sociales, como una de las áreas más diversas y complejas del mundo académico por la diversidad de literatura y la complejidad de la misma, justifica el desarrollo del pensamiento crítico como una manera de valorar rigurosamente la veracidad de los fundamentos y postulados teóricos de cada disciplina.

Pensamiento Crítico y Lectura en Ciencias Sociales

Jesús A. Morales-Carrero

DOI : http://dx.doi.org/10.22458/caes.v8i2.1943

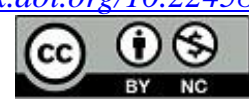


Todo esto equivale a decir que, frente a la abundante información con la que se cuenta en la actualidad, leer en ciencias sociales constituye un reto. Por esta razón, se requiere de un lector acucioso que se valga de múltiples medios para conseguir validar las fuentes, las autorías y los contenidos, con el propósito determinar la veracidad de sus argumentos, de las afirmaciones y de los razonamientos expuestos en los textos científicos. Como consecuencia, se espera que el lector crítico asuma una posición crítica y reflexiva, en la que se cuestione la pertinencia y aplicabilidad del conocimiento a la realidad (Dewey, 1989; Lipman, 1998).

Por otra parte, es oportuno mencionar que dada la interconexión existente entre postulados y fundamentaciones teóricas en ciencias sociales, es necesario abordar procesos de lectura desde una visión sistémica, como una alternativa efectiva que le permita al lector hacer uso de sus modelos teóricos (conocimientos previos) y de los referentes provenientes de varias disciplinas, que articulados con el uso del pensamiento crítico le permitirán la generación de un dialogo con el conocimiento científico que favorezca la construcción reflexiva e interpretativa del nuevas apreciaciones.

En este sentido, el uso del pensamiento crítico como una habilidad superior, enfrenta al lector de las ciencias sociales con el reto de trascender de la mera revisión de ideas y postulados hacia la elaboración de valoraciones significativas, en las que consiga hacer uso competitivo de aspectos como: la caracterización y funcionamiento de los textos, identificación del autor y de su postura ideológica, categoría temática en la que se inscribe, modos y prácticas discursivas utilizadas para comunicar el conocimiento y, la validez, la consistencia y la eficacia de los argumentos que respaldan o sustentan su posición. Entonces, este cúmulo de exigencias requieren,según Girón y otros (2007) de "una actitud y compromiso consigo mismo y con el mundo" (p.46).

Pensamiento Crítico y Lectura en Ciencias Sociales

Jesús A. Morales-Carrero

DOI : http://dx.doi.org/10.22458/caes.v8i2.1943

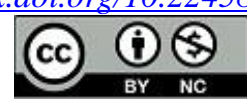


En síntesis, la lectura y el pensamiento crítico como herramientas de aprendizaje (Jurado, 2008; Torres, 2006) representan para el lector la posibilidad de desarrollar una actitud crítica, autónoma y responsable que, fundada en criterios propios le permitan asumir con espíritu reflexivo la objeción, el debate y el cuestionamiento como habilidades cognitivas necesarias para elaborar interpretaciones innovadoras, fundamentadas que definan y re-definan al conocimiento y a las producciones científicas producidas desde diversas comunidades académicas.

\section{REFERENCIAS}

Argudin, \& Luna. M. (1995). Aprendiendo a pensar leyendo bien. En habilidades de lectura a nivel superior. México: Plaza y Valdés Editores.

Aisenberg, B (2007). Ayudar a leer "en sociales". Revista Quehacer educativo, 83, 42-45

Bakalis, M. (2003). Direct teaching of paragraph cohesion. En Teaching History (110): 18

Bartz, W. (2002). Teaching Skepticism via the CRITIC Acronym and the Skeptical Inquirer. En The Skeptical Inquirer, 26(5).

Bazerman, Charles (1988). The problem of writing knowledge. En Shaping writte knowledge. The genre and activity of the experimental article in science, Estados Unidos: The University of Wisconsin Press, 4-17.

Becker, H. (2011). Manual de escritura para científicos sociales: Cómo empezar y terminar una tesis. Buenos Aires: Siglo Veintiuno Editores.

Benavides, D. y Sierra, G. (2013). Estrategias didácticas para fomentar la lectura crítica desde la perspectiva de la transversalidad, 11(03). Recuperado el 05-01-2017 de: http://www.rinace.net/reice/numeros/arts/vol11num3/art4.pdf

Carrasco, A y Kent, R. (2011). Leer y escribir en el doctorado o el reto de formarse como autor de ciencias. Revista Mexicana de Investigación Educativa,16(51), 1227-1251

Pensamiento Crítico y Lectura en Ciencias Sociales

Jesús A. Morales-Carrero

DOI : http://dx.doi.org/10.22458/caes.v8i2.1943

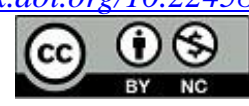


Carlino, P. (2003). Leer textos científicos y académicos en la educación superior: obstáculos y bienvenidas a una cultura nueva. Trabajo presentado en el 60 Congreso internacional de la Promoción de la Lectura y el libro. Buenos Aires, mayo 2003

Carlino, P. (2011). Leer y escribir en las ciencias sociales en universidades argentinas. Revista contextos de educación. Recuperado el 10-02-2017 de: www.hum.unrc.edu.ar/publicaciones/contextos

Cassany, D. (2004). Explorando las necesidades actuales de comprensión aproximaciones a la comprensión. crítica1. Recuperado el 22-02-2017 de: http://www.lecturayvida.fahce.unlp.edu.ar/numeros/a25n2/25_02_Cassany.pdf

Cassany, D. (2006). Tras las Líneas. Sobre la lectura contemporánea. Barcelona: Ediciones Anagrama.

Cassany, D. (2009). 10 claves para enseñar a interpretar. Recuperado el 13-03-2017 de: http://docentes.leer.es/2009/06/25/10-claves-para-ensenar-a-interpretar-docentesdaniel-cassany

Delors, J. (1996). "Los cuatro pilares de la educación" en La educación encierra un tesoro. Informe a la UNESCO de la Comisión internacional sobre la educación para el siglo XXI, Madrid, España: Santillana/UNESCO.

Dewey, J. (1989). ¿Cómo pensamos? Nueva exposición de la relación entre el pensamiento reflexivo y proceso educativo. México: Paidós

Díaz, P., Bar, A. y Ortiz, M. (2015). La lectura crítica y su relación con la formación disciplinar de estudiantes universitarios. Revista de la Educación Superior. XLIV(4), 176, 139-158.

Fortes J. y Lomnitz L. (1991). La formación del científico en México: adquiriendo una nueva identidad. Ciudad de México: Siglo XXI Editores.

González, B y Vega, V. (2010). Prácticas de lectura y escritura en la universidad. El caso de cinco asignaturas de la Universidad Sergio Arboleda. Bogotá D: Escuela de Filosofía y Humanidades.

Goodman, K. (1996). La lectura, la escritura y los textos escritos: Una perspectiva transaccional sociopsicolingüística. En: Textos en contexto. Los procesos de lectura y escritura. Buenos Aires: Asociación Internacional de Lectura

Pensamiento Crítico y Lectura en Ciencias Sociales

Jesús A. Morales-Carrero

DOI : http://dx.doi.org/10.22458/caes.v8i2.1943

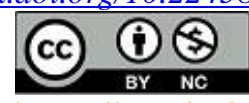


Guerrero, L. y Esquivel, M. (2011). Lectura y escritura. Aprendizaje autónomo: una guía para la docencia. México: Universidad Iberoamericana.

Hammer, D. (1994). Epistemological beliefs in introductory Physics. Cognition and Instruction, $12,151-183$.

Hawes, H. (2003). Pensamiento crítico en la formación universitaria. Universidad de Talca. Chile: Instituto de Investigación y Desarrollo Educacional

Jurado, F. (2008). Formación de lectores críticos desde el aula. Revista Iberoamericana de educación 46, 89-105.

Kock, A. (1991). Improvement of Reading Comprehension of Physics Texts by Students' Question Formulation, En International Journal of Science Education,13, 473-485.

Kurland, D. (2003). Lectura crítica versus pensamiento crítico. Cali: Eduteka.

Lerner, D y et al. (2011). La lectura y la escritura en la enseñanza de Ciencias Naturales y de Ciencias Sociales. Una investigación en didácticas específicas. Anuario de Investigaciones en Ciencias de la Educación, 529-541

Lipman, M. (1997). Pensamiento complejo y educación. Madrid: Ediciones de la Torre.

Menéndez, U. (s/f). El lenguaje jurídico del siglo XXI. Editorial: Uría Menéndez

Morín, E. (2007). El paradigma de complejidad. Introducción al pensamiento complejo. Barcelona: Gedisa.

Newmann, F. (1990). Higher order thinking in the teaching of social studies: Connections between theory and practice. nassp Bulletin, May, 58-64.

Olson, D. (1994). El mundo sobre el papel: el impacto de la escritura y la lectura en la estructura del conocimiento. Barcelona: Gedisa

Pagès, J. (2009). "Enseñar y aprender ciencias sociales en el siglo XXI: reflexiones casi al final de una década". Investigación en Educación, Pedagogía y Formación Docente. Universidad pedagógica nacional, Universidad de Antioquia, Corporación interuniversitaria de servicios, 140-154

Pensamiento Crítico y Lectura en Ciencias Sociales

Jesús A. Morales-Carrero

DOI : http://dx.doi.org/10.22458/caes.v8i2.1943

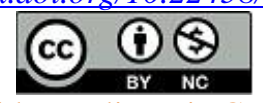


Peppino, M. (2006). Lectura y pensamiento críticos: Estrategias para desenvolverse en el ciberespacio. Universidad Autónoma de México.

Pérez, M. (2005). Evaluación de la comprensión lectora: dificultades y limitaciones. Revista de Educación, número. extraordinario 2005, 121-138

Potapouchkine, N. y Haenisch, H. (2003). La terminología jurídica: dificultades y estrategias de traducción. Revista de la Facultad de Ciencias Jurídicas, 8/9, 205-223

Reale, A. (2016). Leer y escribir textos de Ciencias Sociales: Procesos y estrategias. Universidad Nacional de Quilmes. Argentina: Publicaciones Ciencias Sociales

Sanmarti, N. (2011). Leer para aprender ciencias. Recuperado el 10-01-2017 de: http://leer.es/documents/235507/242734/art_prof_eso_leerciencias_neussanmarti.pdf/b 3507413-ca58-4a00-bf37-c30c619b627f

Santelices, L. (s/f). Desarrollo del pensamiento crítico: su relación con la comprensión de la lectura y otras áreas del currículo de educación básica. Proyecto DIUC 160/82. Santiago, Chile: Pontificia Universidad Católica de Chile.

Santiuste, V. (2001). Quelques réflexions sur la valur éducative de la Philosophie. Conferencia presentada en las Escuelas Europeas. Seminario de Filosofía. Bruselas, Bélgica.

Serna, J. \& Díaz, J. (2014). Propuesta didáctica para la comprensión crítica en la Universidad La Gran Colombia. Cuadernos de Lingüística Hispánica, 25, 165-180.

Serrano, S. (2008). El desarrollo de la comprensión crítica en los estudiantes universitarios: hacia una propuesta didáctica, № 42, 505-514

Smith, E. y Anderson, C. (1993). Teaching strategies associated with conceptual change learning in science. Journal of Research in Science Teaching, 30, 111-126.

Smith, F. (1983). Comprensión de la lectura. Trillas. México.

Solé, I. (1997). Estrategias de lectura. Barcelona: Editorial GRAÓ.

Pensamiento Crítico y Lectura en Ciencias Sociales

Jesús A. Morales-Carrero

DOI : http://dx.doi.org/10.22458/caes.v8i2.1943

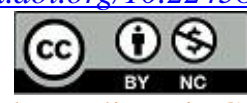


Torres, R. (2006). Alfabetización y aprendizaje a lo largo de toda la vida. Instituto Fronesis. Recuperado el 10-02-2017 de: www.oei.es/alfabetizacion/AprendizajePermanenteESP.pdf

Ulloa, J; Ulloa., Crispín, L y Bejár, M. (s/f). La lectura y la escritura ¿se deben aprender en la universidad? Programa de Formación de Académicos. № 10, 1-7 